\title{
Error estimation for localized signal properties: application to atmospheric mixing height retrievals
}

\section{G. Biavati et al.}

Correspondence to: G. Biavati (gbiavati@bgc-jena.mpg.de)

The copyright of individual parts of the supplement might differ from the CC-BY 3.0 licence. 
Here we present the application for the described methodology with simple numerical examples. We will focus on two cases: the detection of a local maximum and a threshold detection. For the first case, we use a Gaussian curve $g(x)$ as the test function. For the second case, we use the integral of a Gaussian curve function $e(x)$ as plotted in Fig. S1. We use the function $g(x)$ in order to estimate the local maximum, which corresponds to the global maximaum. For this, we use a very simple algorithm: chose the first occurence of the absolute maximum in the dataset.

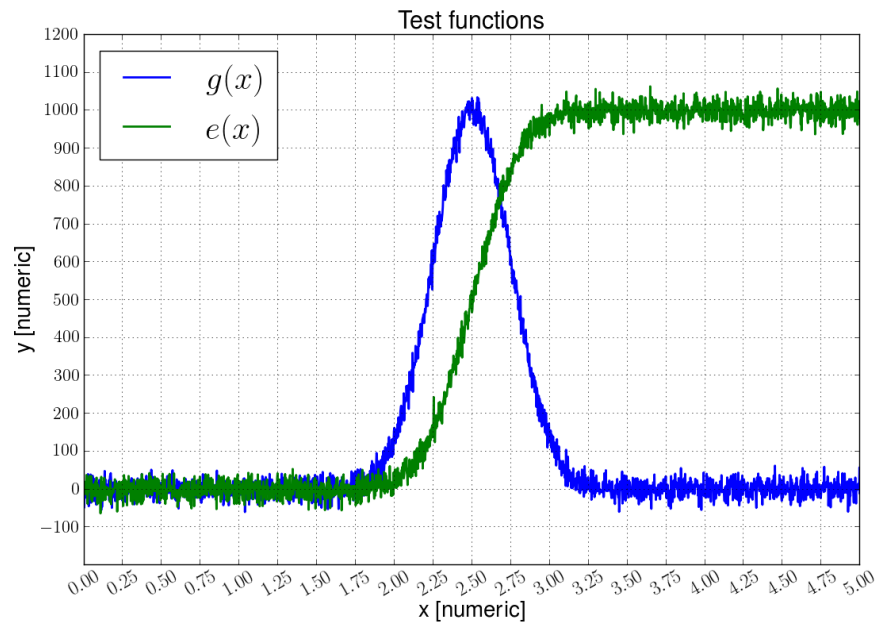

Figure S1: Two examples of curves used, a Gaussian curve $g(x)$ and and a sigmoid function $e(x)$ set to have maximum value equal to 1000 and with applied normal noise of standard deviation $=20$.

We performed a simple Monte Carlo (MC) approach to justify that the results are consistent with our method's results at the suggested confidence level of $\gamma=2$. We modified several parameters from the curve's slope to the noise intensity. The results are presented in Fig. S2. On the left column, the results for $g(x)$ and on the right column those for $e(x)$. Each point in the result is produced after 1000 iterations. The algorithm used to retrieve the local maximum on $g(x)$ is the one described before (first occurence of the global maximum). The algorithm used on $e(x)$ is to locate the first point whose value is equal or greater that 500 .

From the plot, it is reasonable to say that $\sigma_{x_{m}}$ obtained using confidence neighborhoods is comparable to the distribution's standard deviation of results obtained by iterating the algorithm after applying noise. In particular for the second column, which corresponds to the results of a threshold reached on a well defined slope, the value of $\gamma=2$ shows best agreement between $\sigma_{x_{m}}$ obtained using confidence neighborhoods and the classical standard deviation using a Monte Carlo approach.

Several other numerical experiment where made with different curves and noise intensities. All showed similar results, so $\gamma=2$ is the value we suggest.
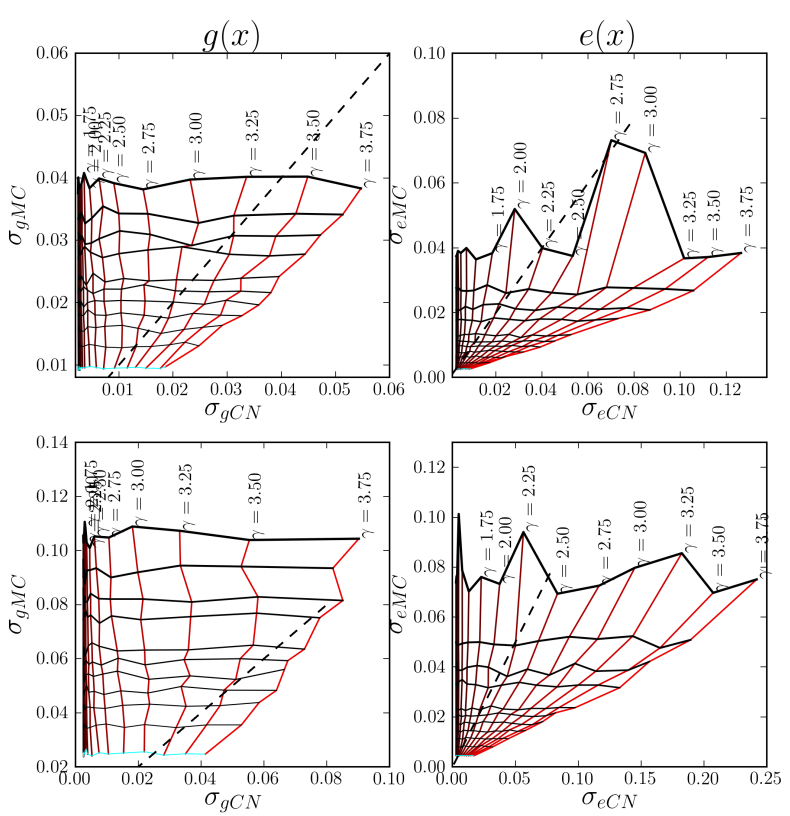

Figure S2: Results of the Monte Carlo. First column presents the results for $g(x)$, the second for $e(x)$. In the first row curves are more steep, the Gaussian curve is calculated with a standard deviation of 0.25 . In the second row the curves are more gentle with the standard deviation of the Gaussian curve set to 0.5. The horizontal axes shows the average errors obtained using our methodology. The vertical axes shows the standard deviation of the location obtained using the algorithms. Almost horizontal lines define the noise applied to the curves starting from $0.5 \%$ to $10 \%$ of the maximum value which is 1000 . The almost vertical lines represent different $\gamma$ thresholds used for the calculation of $U_{\gamma}\left(x_{m}\right)$. The dashed line is the identity. 